\title{
Homotopy analysis method for solving MHD free convec- tion flow from a cooling sheet
}

\author{
Zakia Hammouch ${ }^{\mathrm{a}, *}$, Toufik Mekkaoui ${ }^{\mathrm{a}}$, Hssain Sadkib \\ ${ }^{a}$ E3MI, Département de mathématique, FST Errachidia, Université Moulay Ismail, BP. 509, Boutalamine, 52000 Errachidia, Morocco. \\ ${ }^{b}$ Département d'Informatique, FST Errachidia, Université Moulay Ismail, BP. 509, Boutalamine, 52000 Errachidia, Morocco.
}

\begin{abstract}
In this paper, we investigate the problem of MHD free convection cooling of a low-heat-resistance sheet that moves downwards in a viscous fluid. The basic equations are converted into coupled ordinary differential equations via the similarity transformation, and solved analytically using homotopy analysis method (HAM). The obtained analytical solutions for both of the velocity and the temperature with different values of the Prandtl number $\mathrm{Pr}$ and the magnetic parameter $\mathrm{M}$ are plotted and discussed in detail.
\end{abstract}

Keywords: Homotopy analysis method, cooling, MHD flow, similarity solution.

2010 MSC: 35C10, 76W05, 76M55, 80A20.

(C)2018 All rights reserved.

\section{Introduction}

Convective flows in porous media have a wide range of applications in engineering and science. Examples of these applications include solar energy systems, boilers, cooling of electronic devices, compact heat exchangers and the cooling core of nuclear reactors (see [3, 13, 15, 17, 21, 24]). Furthermore, Magnetohydrodynamics (MHD) which is the study of the interaction of conducting fluids with electromagnetic phenomena, is of a great importance due to its applications in various areas of technology and engineering such as designing cooling systems which are liquid metals, MHD generators, accelerators, pumps and flow meters.

The homotopy analysis method (HAM), proposed by Liao in his $\mathrm{PhD}$ thesis [28], is an analytic tool for handling nonlinear problems, it can be considered as one of the powerful method for solving nonlinear problems since it yields rapidly convergent series solutions in most cases, which lead usually to very accurate solutions only in few iterations. In recent years, this method has been successfully used to solve many types of nonlinear problems in science and engineering such as the magnetohydrodynamic flows of non-Newtonian fluids over a stretching sheet [1], boundary-layer flows over an impermeable

\footnotetext{
*Corresponding author

Email addresses: z.hammouch@fste.umi.ac.ma (Zakia Hammouch), t.mekkaoui@fste.umi.ac.ma (Toufik Mekkaoui),

s.hssain@fste.umi.ac.ma (Hssain Sadki)
}

doi: $10.22436 / \mathrm{mns} .03 .01 .05$

Received: 2016-10-31 Revised: 2017-02-14 Accepted: 2017-07-24 
stretched plate [2], nonlinear model of combined convective and radiative cooling of a spherical bodies, exponentially decaying boundary layers [9], unsteady boundary-layer flows over a stretching flat plate [10]. Also, the boundary-layer flow problem was considered by Ghotbi et al. [12] and Hashim et al. [24]. The present paper extends the results of previous authors by considering the effect of the magnetic field on the velocity and the temperature on velocity slip. The method we employed here is based on the homotopy analytical method (HAM).

More recently, Bataineh et al. [5] applied the standard HAM to solve some problems in engineering sciences. All of these successful applications verified the validity and the effectiveness of the HAM.

In this paper we aim to find the analytical solutions for the flow problem considered in [12, 24]. Analytic solutions for the velocity and temperature components are obtained using a powerful, east-touse technique, namely the homotopy analysis method (HAM). The series solution is firstly computed and then its convergence is properly discussed. Finally, the graphs are plotted and discussed for the variation of Prandtl numbers and Hartmann numbers. The method gives rapidly convergent series with specifics significant features for each scheme.

\section{Formulation of the problem}

Consider the problem of cooling of a low-heat-resistance sheet that moves downwards in a viscous fluid in presence of a magnetic field. This phenomenon can be modeled by (cf. $[3,11,26])$

$$
\left\{\begin{array}{l}
\frac{\partial u}{\partial x}+\frac{\partial v}{\partial y}=0 \\
u \frac{\partial u}{\partial x}+v \frac{\partial u}{\partial y}=v \frac{\partial^{2} u}{\partial y^{2}}+g \beta\left(T-T_{0}\right)-\frac{\sigma B^{2} x}{\rho} u \\
u \frac{\partial T}{\partial x}+v \frac{\partial T}{\partial y}=\kappa \frac{\partial^{2} T}{\partial y^{2}}
\end{array}\right.
$$

with the conditions

$$
\begin{cases}u(x, 0)=0, & v(x, 0)=0, \\ \mathfrak{u}(x, \infty)=0, & \mathrm{~T}(x, \infty)=\mathrm{T}_{0},\end{cases}
$$

where $u$ and $v$ are the velocity components in the $x$ and $y$ directions, respectively, $T$ is the temperature, $T_{0}$ is the temperature of the surrounding fluid, $v$ is the kinematic viscosity, $k$ is the thermal diffusivity, $g$ is the acceleration due to gravity, and $\beta$ is the coefficient of thermal expansion.

Using the similarity variables:

$$
\left\{\begin{array}{l}
\eta(x, y)=\left(\frac{g \beta\left(T_{1}-T_{0}\right) x_{0}^{3}}{v^{2}}\right)^{1 / 4}\left(\frac{y}{x_{0}-x}\right), \\
\psi(x, y)=\left(g \beta\left(T_{1}-T_{0}\right) v^{2} x_{0}^{3}\right)^{1 / 4} f(\eta), \\
T(x, y)=T_{0}+\left(T_{1}-T_{0}\right)\left(\frac{x_{0}}{x_{0}-x}\right)^{3} \theta(\eta),
\end{array}\right.
$$

Where $\psi$ is the stream function defined by $u=\frac{\partial \psi}{\partial y}$ and $v=-\frac{\partial \psi}{\partial y}$, $f$ and $\theta$ are the dimensionnless stream function and temperature, respectively, and $T(0,0)=T_{1}$. Problem (2.1)-(2.2) is transformed to the following

$$
\left\{\begin{array}{l}
\theta^{\prime \prime}=3 \operatorname{Prf}^{\prime} \theta \\
f^{\prime \prime \prime}-f^{\prime 2}-M f^{\prime}+\theta=0
\end{array}\right.
$$

subject to the boundary conditions

$$
\begin{cases}f(0)=0, & f^{\prime}(0)=0, \quad f^{\prime}(\infty)=0 \\ \theta(0)=1, & \theta(\infty)=0,\end{cases}
$$


where the prime is for differentiation with respect to the similarity variable $\eta, \operatorname{Pr}=\frac{v}{\kappa}$, is the Prandtl number, $B(x)=\frac{\sigma}{\rho} B_{0}^{2} \frac{\left(g \beta\left(T_{1}-T_{0}\right) x_{0}^{3}\right)^{1 / 2}}{\left(x_{0}-x\right)^{2}}$ is the magnetic field, and $M=\frac{\sigma}{\rho} B_{0}^{2}$ is the Hartmann number.

In the remainder of this paper, we shall solve this two-point boundary value problem analytically using a Homotopy analysis method.

\section{The HAM solutions of the problem}

In this section, we apply the homotopy analysis method to solve the system (2.3)-(2.4). The first step in the solution process is to choose a proper initial guess approximations satisfying the boundary conditions and to find the linear operator. For the present problem, we choose the initial guess approximations for velocity and temperature as follows

$$
\left\{\begin{array}{l}
\theta_{0}(\eta)=e^{-\eta} \\
f_{0}(\eta)=0
\end{array}\right.
$$

Besides, we select the auxiliary linear operators $L_{1}$ and $L_{2}$ for velocity and temperature functions, respectively, of the form

$$
\left\{\begin{array}{l}
L_{1}(f)=f^{\prime \prime \prime}-f^{\prime} \\
L_{2}(\theta)=\theta^{\prime \prime}-\theta
\end{array}\right.
$$

satisfying the following properties

$$
\left\{\begin{array}{l}
\mathrm{L}_{1}\left(\mathrm{C}_{1}+\mathrm{C}_{2} e^{\eta}+\mathrm{C}_{3} e^{-\eta}\right)=0 \\
\mathrm{~L}_{2}\left(\mathrm{C}_{4} e^{\eta}+\mathrm{C}_{5} e^{-\eta}\right)=0
\end{array}\right.
$$

where $C_{i}, i=1, \ldots, 5$.

\subsection{Zeroth-order deformation problems}

First we construct the zeroth-order deformation problem as the following

$$
\left\{\begin{array}{l}
(1-p) L_{1}\left[f(\eta ; p)-f_{0}(\eta)\right]=p \hbar_{1} N_{1}[f(\eta ; p), \theta(\eta ; p)] \\
f(0 ; p)=0, \quad f^{\prime}(0 ; p)=0, \quad f^{\prime}(\infty ; p)=0 \\
(1-p) L_{2}\left[\theta(\eta ; p)-\theta_{0}(\eta)\right]=p \hbar_{2} N_{2}[f(\eta ; p), \theta(\eta ; p)] \\
\theta(0 ; p)=1, \quad \theta(\infty ; p)=0
\end{array}\right.
$$

where

$$
\left\{\begin{array}{l}
N_{1}[f(\eta ; p), \theta(\eta ; p)]=\frac{\partial^{3} f(\eta ; p)}{\partial \eta^{3}}+\theta(\eta ; p)-M \frac{\partial f(\eta ; p)}{\partial \eta}-\left(\frac{\partial f(\eta ; p)}{\partial \eta}\right)^{2}, \\
N_{2}[f(\eta ; p), \theta(\eta ; p)]=\frac{\partial^{2} \theta(\eta ; p)}{\partial \eta^{2}}-3 \operatorname{Pr} \frac{\partial f(\eta ; p)}{\partial \eta} \theta(\eta ; p),
\end{array}\right.
$$

where $p \in[0,1]$ is the so-called embedding parameter, $\hbar_{1}$ and $\hbar_{2}$ are the non-zero auxiliary parameter. Notice that $p=0$ and $p=1$ give

$$
f(\eta ; 0)=f_{0}(\eta) \text { and } \theta(\eta ; 0)=\theta_{0}(\eta) .
$$

Where $f(\eta ; p)$ (resp. $\theta(\eta ; p)$ ) varies from the known initial approximation $f_{0}(\eta ; p)$ (resp. $\theta_{0}(\eta ; p)$ ) to the unknown solution $f(\eta)$ (resp. $\theta(\eta)$ ) when the embedding-parameter $p$ moves from 0 to 1 .

Expanding $f(\eta ; p)$ and $\theta(\eta ; p)$ in Taylor series with respect to the embedding parameter $q$, we get

$$
\left\{\begin{array}{l}
f(\eta ; p)=f_{0}(\eta)+\sum_{m=1}^{\infty} f_{m}(\eta) p^{m}, \quad f_{m}(\eta)=\frac{1}{m !} \frac{\partial^{m} f(\eta ; p)}{\partial p^{m}} \\
\theta(\eta ; p)=\theta_{0}(\eta)+\sum_{m=1}^{\infty} \theta_{m}(\eta) p^{m}, \quad \theta_{m}(\eta)=\frac{1}{m !} \frac{\partial^{m} \theta(\eta ; p)}{\partial p^{m}} .
\end{array}\right.
$$


Note that the auxiliary parameters $\hbar_{1}$ and $\hbar_{2}$ are properly chosen in such a way that these two series are convergent series (3.2) are convergent at $p=1$. Therefore we have through equation (3.2)

$$
f(\eta)=f_{0}(\eta)+\sum_{m=1}^{\infty} f_{m}(\eta), \quad \theta(\eta)=f_{0}(\eta)+\sum_{m=1}^{\infty} \theta_{m}(\eta) .
$$

3.2. $\mathrm{m}^{\text {th }}$-order deformation problems

Differentiating the zeroth-order deformation equations (3.1) m-times with respect to $p$, then setting $p=0$, and finally dividing them by $m !$, we obtain the $m^{\text {th }}$-order deformation equations

$$
\left\{\begin{array}{l}
L_{1}\left[f_{m}(\eta)-\chi_{m} f_{m-1}(\eta)\right]=\hbar_{1} R_{m}(\eta) \\
f_{m}(0)=f_{m}^{\prime}(0)=f_{m}^{\prime}(\infty)=0 \\
L_{2}\left[\theta_{m}(\eta)-\chi_{m} \theta_{m-1}(\eta)\right]=\hbar_{2} Q_{m}(\eta) \\
\theta_{m}(0)=\theta_{m}(0)=0
\end{array}\right.
$$

and

$$
\left\{\begin{array}{l}
\mathrm{R}_{\mathrm{m}}=\mathrm{f}_{\mathrm{m}-1}^{\prime \prime \prime}+\theta_{\mathrm{m}-1}-\sum_{n=0}^{\mathrm{m}-1} \mathrm{f}_{\mathrm{m}-1-\mathrm{n}}^{\prime} \mathrm{f}_{\mathrm{n}}^{\prime} \\
\mathrm{Q}_{\mathrm{m}}=\theta_{m-1}^{\prime \prime}-3 \operatorname{Pr} \sum_{n=0}^{m-1} f_{m-1-n}^{\prime} \theta_{n}-M f_{m-1}^{\prime},
\end{array} \quad \chi_{m}= \begin{cases}0, & m \leqslant 1 \\
1, & m>1\end{cases}\right.
$$

The first two deformations of the coupled solutions are computed using MAPLE software, we obtain the following solutions of problem (2.3)-(2.4) for the Hrtmann number $M=0.5$ and the Prandtl $\operatorname{Pr}=1$.

$$
\left\{\begin{aligned}
\bullet f_{1}(\eta)= & -0.1666666667 \frac{h}{e^{2 t}}+1.416118082 \times 10^{-18} h e^{t}+0.3333333333 \frac{h}{e^{t}}-0.1666666667 h, \\
\bullet \theta_{1}(\eta)= & 0.3333333333 \frac{h^{2 t}}{e^{2 t}}+3.119206702 \times 10^{-14} h e^{t}-0.3333333334 \frac{h^{t}}{e^{t}}, \\
\bullet f_{2}(\eta)= & -0.1666666667 \frac{h}{\left(e^{t}\right)^{2}}+1.416118082 \times 10^{-18} h e^{t}+0.3333333334 \frac{h}{e^{t}}-0.1666666667 h \\
& -1.559638754 \times 10^{-14} h^{2} t-0.06250000000 \frac{h^{2}}{\left(e^{t}\right)^{3}}-1.559638754 \times 10^{-14} h^{2} \ln \left(e^{t}\right), \\
& +0.08333333332 \frac{h^{2}}{\left(e^{t}\right)^{2}}+0.02083333340 \frac{h^{2}}{e^{t}}+8.857167512 \times 10^{-20} h^{2} e^{t}-0.04166666672 h^{2} \\
\bullet \theta_{2}(\eta)= & 0.3333333333 \frac{h}{\left(e^{t}\right)^{2}}+3.119206702 \times 10^{-14} h e^{t}-0.3333333333 \frac{h}{e^{t}}+0.2916666664 \frac{h^{2}}{\left(e^{t}\right)^{3}} \\
& -3.119206702 \times 10^{-14} h^{2}-0.1138888885 \frac{h^{2}}{e^{t}}+2.124177123 \times 10^{-18} \frac{h^{2} t}{e^{t}}-0.1111111112 \frac{h^{2}}{\left(e^{t}\right)^{2}} \\
& -0.06666666670 \frac{h^{2}}{\left(e^{t}\right)^{4}}+1.065730551 \times 10^{-14} h^{2} e^{t} .
\end{aligned}\right.
$$

The solutions $f_{i}(\eta)$ and $\theta_{i}(\eta)$ for $i \geqslant 3$ were too long to be mentioned here, therefore, they are shown graphically. But it is easy to compute them by using Maple software.

\section{Convergence of the HAM solutions}

According to Liao [29], the convergence and the rate of approximation for the HAM solution is strongly dependent on the values of the auxiliary parameter $h$. Figures 1 and 3 show the $h$-curves of $f^{\prime \prime}(0)$ at different orders of approximations, for various values of the Hartmann number $M$ and the Prandtl number Pr, respectively. We remark that the convergence is strongly dependent on the values of involving parameters. 
4.1. Effect of the Hartman number $M$
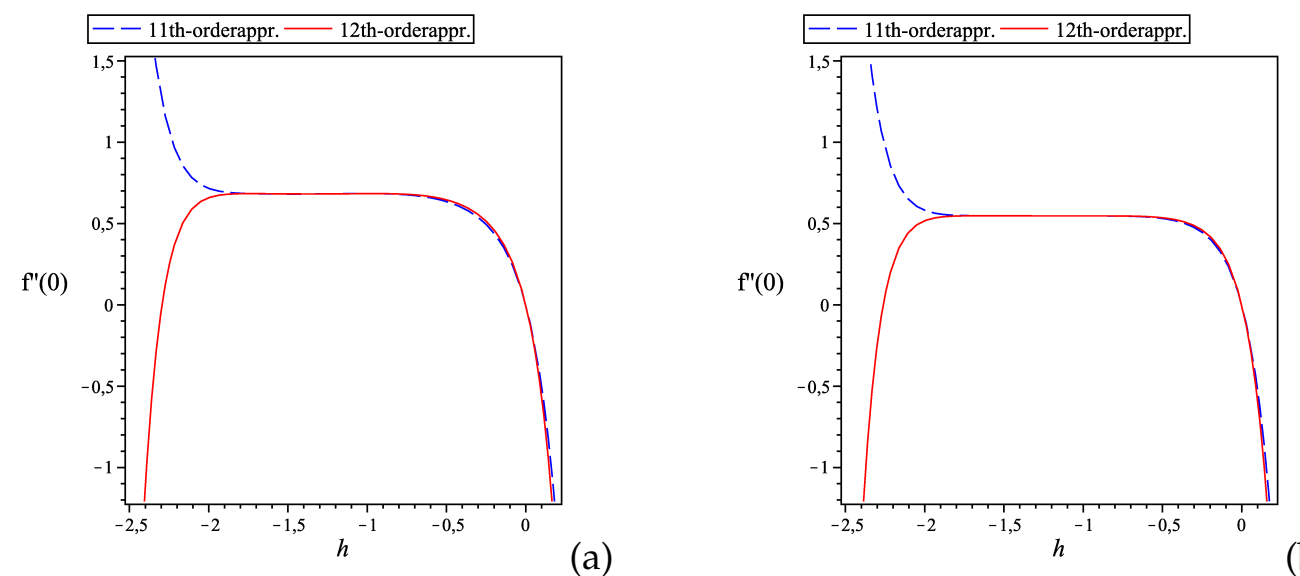

(a)

(b)

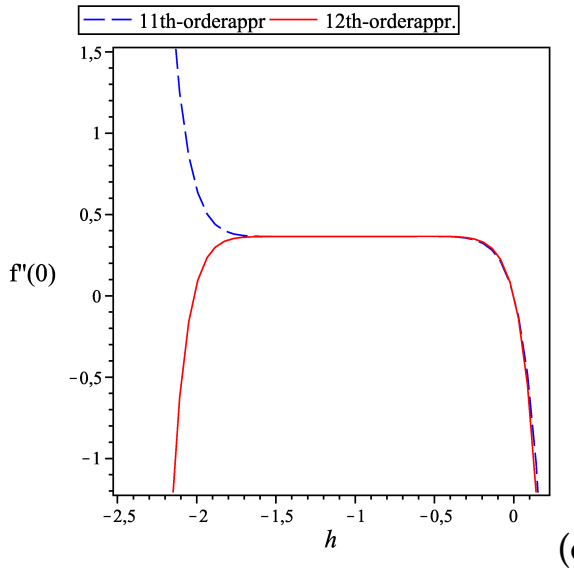

(c)

Figure 1: h-curves of $f^{\prime \prime}(0)$ at different orders of approximations, when: (a) $M=0, \operatorname{Pr}=1$. (b) $M=1, \operatorname{Pr}=1$, and (c) $M=5$, $\operatorname{Pr}=1$.

Table 1: Variation of the Nusselt number and the skin friction for various values of the Hartmann number and $\operatorname{Pr}=1$.

\begin{tabular}{|c|c|c|}
\hline$M$ & $-\theta^{\prime}(0)$ & $f^{\prime \prime}(0)$ \\
\hline 00.00 & 0.75637 & 0.68176 \\
\hline 01.00 & 0.65517 & 0.54707 \\
\hline 05.00 & 0.47119 & 0.36412 \\
\hline
\end{tabular}
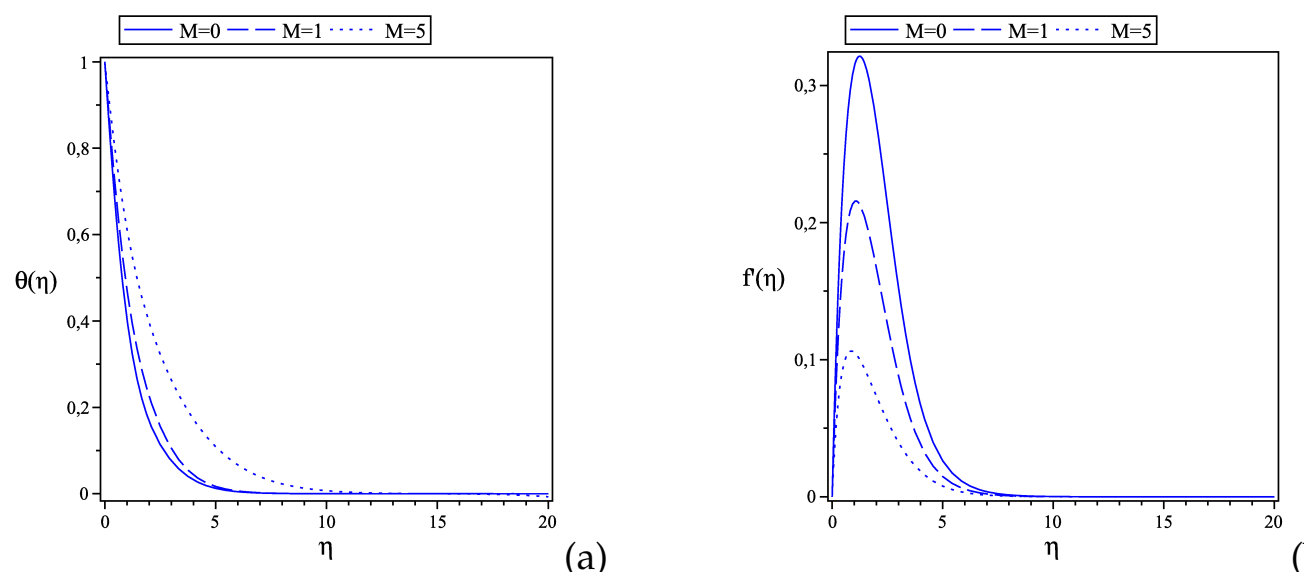

(a)

(b) 


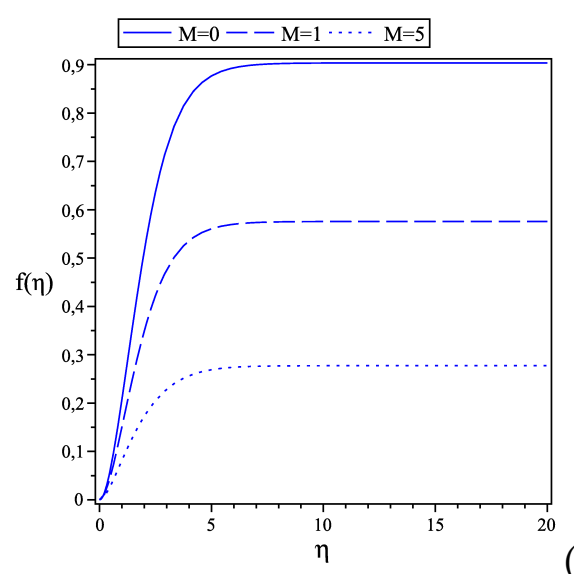

(c)

Figure 2: Effect of the Hartman number on: (a) non-dimensional temperature profiles, (b) non-dimensional velocity, (c) nondimensional stream-function; when $M=0.5$.

\subsection{Effect of the Prandtl number $\mathrm{Pr}$}
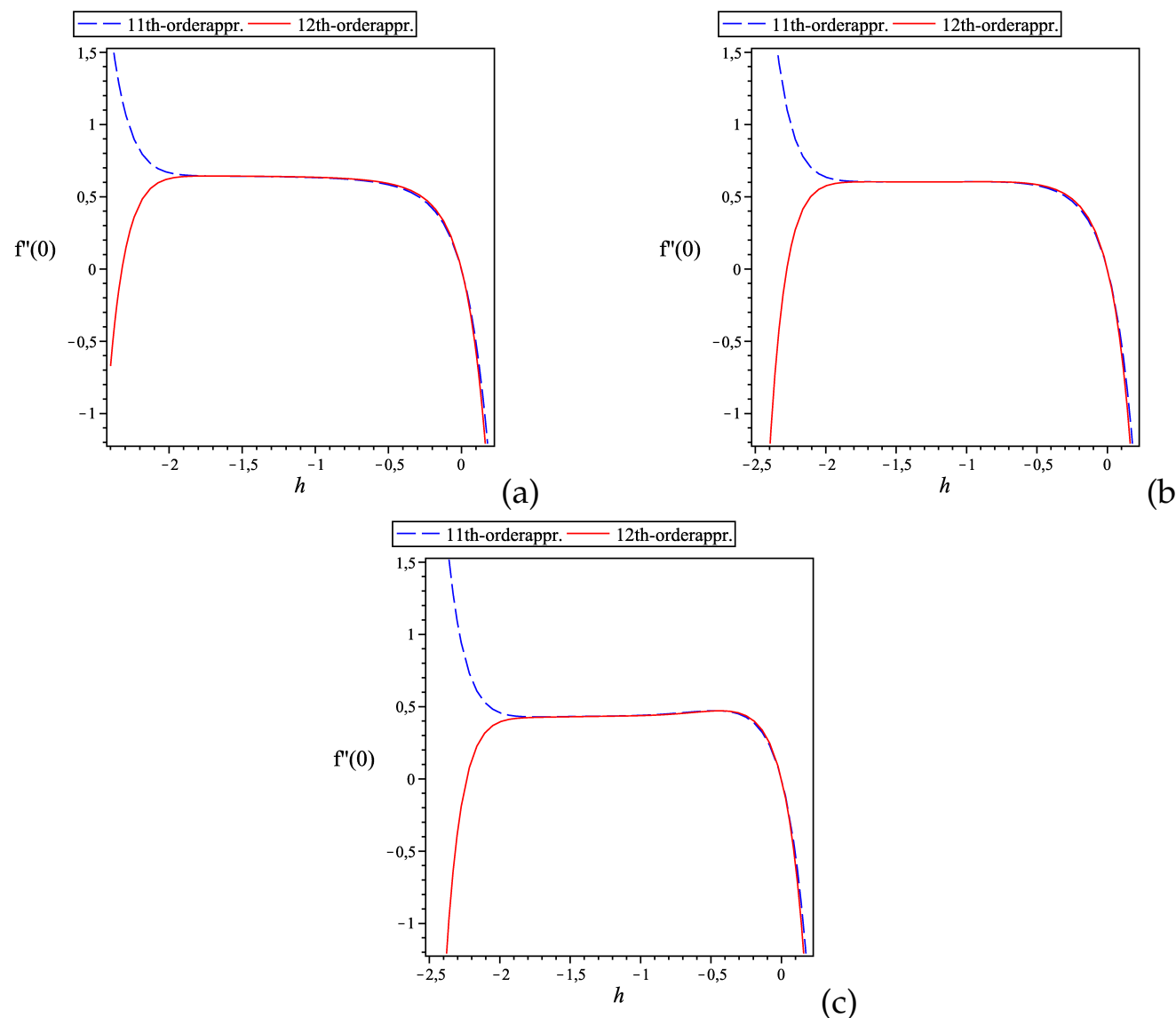

(c)

Figure 3: h-curves of $f^{\prime \prime}(0)$ at different orders of approximations, when: (a) $M=0.5, \operatorname{Pr}=0.1$. (b) $M=0.5, \operatorname{Pr}=1$, and (c) $M=0.5, \operatorname{Pr}=10$. 

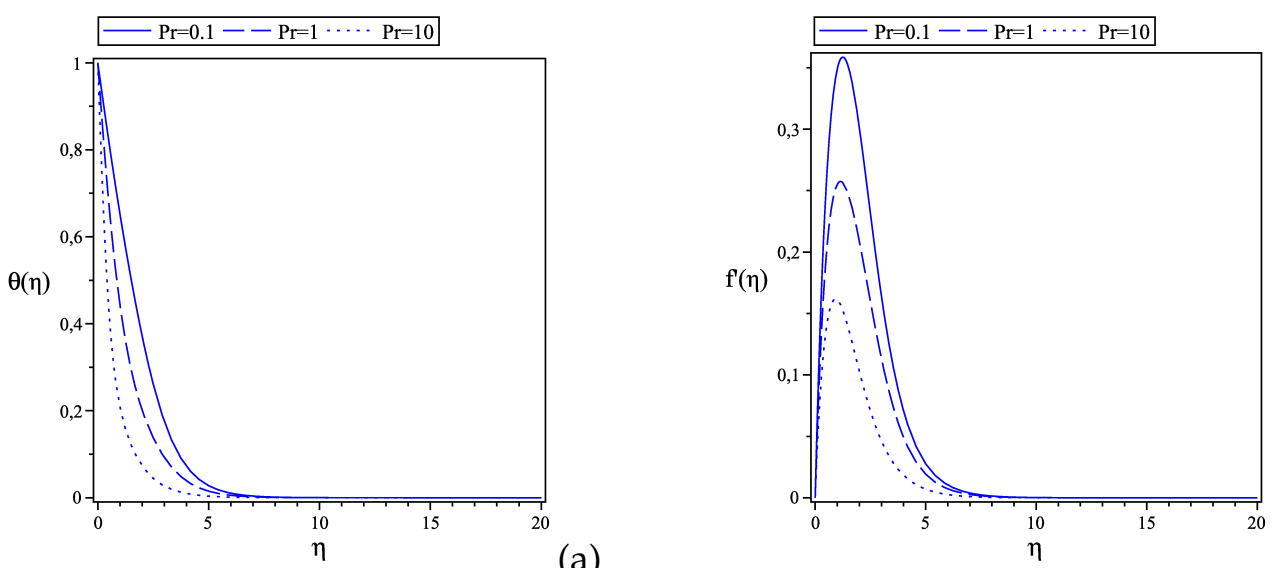

(a)

(b)

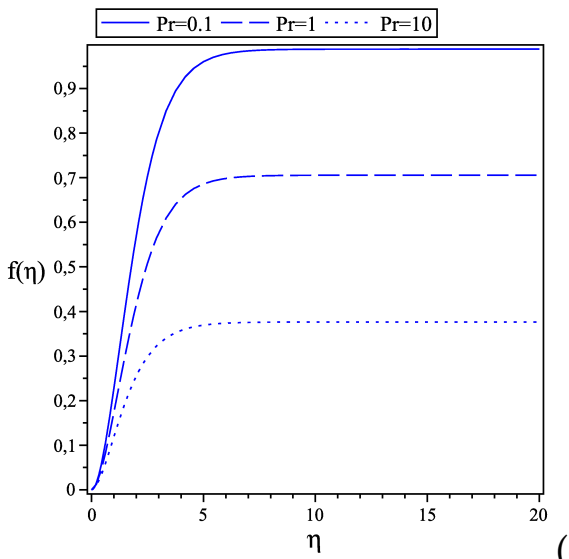

(c)

Figure 4: Effect of the Prandtl number on: (a) non-dimensional temperature profiles, (b) non-dimensional velocity, (c) nondimensional stream-function; when $M=0.5$.

Table 2: Variation of the Nusselt number and the skin friction for various values of the Prandtl number and $M=0.5$.

\begin{tabular}{|c|c|c|}
\hline $\operatorname{Pr}$ & $-\theta^{\prime}(0)$ & $f^{\prime \prime}(0)$ \\
\hline 0.10 & 0.36906 & 0.72711 \\
\hline 1.00 & 0.69841 & 0.60294 \\
\hline 10.0 & 1.42065 & 0.42965 \\
\hline
\end{tabular}

\section{Summary and conclusion}

A comprehensive computation is conducted for various physical parameters involved in current problem. To determine the accuracy in favor of present results, a comparison of obtained results for the skin friction and local Nusselt number are presented in Tables. It is seen from Table 1 that the increase in the Hartmann number decreases the skin-friction coefficient, which means the magnetic field retard the flow and reduces the flow velocity. Besides, increasing Hartmann numbers decreases the Nusselt number (rate of heat transfer), the reason of this is retarding effect of magnetic field on convection . On the other hand, it is observed from Fig. 2 that as magnetic parameter increases, the fluid velocity decreases. However, the fluid temperature increases with the increase in the magnetic parameter. So the magnetic field can therefore be used to control the flow characteristics. Table 2 and Figure 3 show the increase in Prandtl number causes reduction in the temperature gradient (i.e., $\left.\theta^{\prime}(0)\right)$ at the surface of the plate. The temperature gradient reduces at the surface because low Prandtl fluid has high thermal conductivity, causing the fluid to attain higher temperature thereby reducing heat flux at the surface. Also, for such low Prandtl number, the velocity boundary layer is inside the thermal boundary layer and its thickness reduces as 
Prandtl number decreases so the fluid motion is confined in more and more thinner layer near surface, which experiences increased drag (skin-friction) by the fluid. Thus higher Prandtl number leads to faster cooling of the plane sheet.

\section{References}

[1] S. Abbasbandy, The application of homotopy analysis method to nonlinear equations arising in heat transfer, Phys. Lett. A, 360 (2006), 109-113. 1

[2] S. Abbasbandy, Homotopy analysis method for heat radiation equations, Int. Commun. Heat Mass Transfer, 34 (2007), 380-387. 1

[3] F. M. Ali, R. Nazar, N. M. Arifin, Numerical Investigation of Free Convective Boundary Layer in a Viscous Fluid, Amer. J. Sci. Res., 5 (2009), 13-19. 1, 2

[4] M. Amkadni, A. Azzouzi, Z. Hammouch, On the exact solutions of laminar MHD flow over a stretching flat plate, Commun. Nonlinear Sci. Numer. Simul., 13 (2008), 359-368.

[5] A. S. Bataineh, M. S. M. Noorani, I. Hashim, Homotopy analysis method for singular IVPs of EmdenFowler type, Commun. Nonlinear Sci. Numer. Simul., 14 (2009), 1121-1131. 1

[6] N. Bedjaoui, M. Guedda, Z. Hammouch, Similarity solutions of the Rayleigh Problem for ostwald-de Wael electrically conduction fluids, Anal. Appl., 9 (2011), 135-159.

[7] A. Bejan, Convection heat transfer, Wiley, New York, (1995).

[8] G. Domairry, A. Mohsenzadeh, M. Famouri, The application of homotopy analysis method to solve nonlinear differential equation governing Jeffery-Hamel flow, Commun. Nonlinear Sci. Numer. Simul., 14 (2009), 85-95.

[9] G. Domairry, N. Nadim, Assessment of homotopy analysis method and homotopy perturbation method in non-linear heat transfer equation, Int. Commun. Heat Mass Transfer, 35 (2008), 93-102. 1

[10] A. Fakhari, G. Domairry, M. Ebrahimpour, Approximate explicit solutions of nonlinear BBMB equations by homotopy analysis method and comparison with the exact solution, Phys. Lett. A, 368 (2007), 64-68. 1

[11] A. R. Ghotbi, Homotopy analysis method for solving the MHD flow over a non-linear stretching sheet, Commun. Nonlinear Sci. Numer. Simul., 14 (2009), 2653-2663. 2

[12] A. R. Ghotbi, H. Bararni, G. Domairry, A. Barari, Investigation of a Powerful Analytical Method into Natural Convection Boundary Layer Flow, Commun. Nonlinear Sci. Numer. Simul., 14 (2009), 2222-2228. 1

[13] M. Guedda, Z. Hammouch, On similarity and pseudo-similarity solutions of FalknerSkan boundary layers, Fluid Dynam. Res., 38 (2006), 211-223. 1

[14] M. Guedda, Z. Hammouch, Similarity Flow Solutions of a Non-Newtonian Power-law Fluid, Int. J. Nonlinear Sci., 6 (2008), 255-264.

[15] Z. Hammouch, Étude mathématique et numérique de quelques problèmes issus de la dynamique des fluides, Doctoral dissertation, Amiens, (2006). 1

[16] Z. Hammouch, Multiple solutions of steady MHD flow of dilatant fluids, Eur. J. Pure Appl. Math., 1 (2008), 11-20.

[17] Z. Hammouch, M. Guedda, Existence and non-uniqueness of solution for a mixed convection flow through a porous medium, J. Appl. Math. Inform., 31 (2013), 631-642. 1

[18] Z. Hammouch, T. Mekkaoui, F. B. M. Belgacem, Double-diffusive natural convection in porous cavity heated by an internal boundary, Math. Eng. Sci. Aeorospace, 7 (2016), 1-14.

[19] Z. Hammouch, T. Mekkaoui, H. Sadki, Similarity solutions of a steady MHD flow over a semi-infinite surface, Math. Eng. Sci. Aerospace, 8 (2017), 1-9.

[20] R. U. Haq, Z. Hammouch, S. T. Hussain, T. Mekkaoui, MHD mixed convection flow along a vertically heated sheet, Int. J. Hydrogen Energ., 42 (2017), 15925-15932.

[21] R. U. Haq, Z. Hammouch, A. W. Khan, Water-based squeezing flow in the presence of carbon nanotubes between two parallel disks, Thermal Science, 20 (2014), 1973-1981. 1

[22] R. U. Haq, Z. H. Khan, S. T. Hussain, Z. Hammouch, Flow and heat transfer analysis of water and ethylene glycol based Cu nanoparticles between two parallel disks with suction/injection effects, J. Molecular Liquids, 221 (2009), 298-304.

[23] R. U. Haq, F. A. Soomro, Z. Hammouch, Heat transfer analysis of $\mathrm{CuO}$-water enclosed in a partially heated rhombus with heated square obstacle, Int. J. Heat Mass Transfer, 118 (2018), 773-784.

[24] A. Ishak, R. Nazar, I. Pop, MHD boundary-layer flow due to a moving extensible surface, J. Engrg. Math., 62 (2008), 23-33. 1

[25] W. M. Kays, M. E. Crawford, Convection heat and mass transfer, McGraw-Hill, New York, (1993).

[26] S. A. Kechil, I. Hashim, Non-Perturbative Solution of Free-Convective Boundary-Layer Equation by Adomian Decomposition Method, Phys. Lett. A, 363 (2010), 110-114. 2

[27] S. L. Lee, J. S. Tsai, Cooling of a continuous moving sheet of finite thickness in the presence of natural convection, Int. J. Heat Mass Transfer, 33 (1990), 457-464.

[28] S.-J. Liao, On the homotopy Analysis Method for non linear problems, Appl. Math. Comput., 147 (2004), 499-513. 1

[29] S.-J. Liao, A new branch of solutions of boundary layer flows over a permeable stretching plate, Int. J. Non-Linear. Mech., 42 (2007), 819-830. 4 
[30] R. Muthuraj, S. Srinivas, Fully develloped MHD flow of a micropolar and viscous fluids in a vertical porous space using HAM, Int. J. Appl. Math. Mech., 6 (2010), 55-78.

[31] A. Shafiq, Z. Hammouch, T. N. Sindhu, Bioconvective MHD flow of tangent hyperbolic nanofluid with newtonian heating, Int. J. Mech. Sci., 133 (2017), 759-766.

[32] A. Shafiq, Z. Hammouch, A. Turab, Impact of radiation in a stagnation point flow of Walters B fluid towards a Riga plate, Thermal Sci. Eng. Progress, 6 (2017), 27-33. 